EPSC Abstracts

Vol. 15, EPSC2021-413, 2021

https://doi.org/10.5194/epsc2021-413

Europlanet Science Congress 2021

(C) Author(s) 2022. This work is distributed under

the Creative Commons Attribution 4.0 License.

\title{
Characterisation of the hydrodynamic atmospheric escape of HD 209458 b, HD 189733 b, and GJ 3470 b
}

Manuel Lampón ${ }^{1}$, Manuel López-Puertas ${ }^{1}$, Alejandro Sánchez-López ${ }^{2}$, Stefan Czesla ${ }^{3}$, Jorge SanzForcada $^{4}$, and the CARMENES Consortium*

${ }^{1}$ Instituto de Astrofísica de Andalucía, CSIC, Granada, Spain (mlampon@iaa.es)

${ }^{2}$ Leiden Observatory, Leiden University, Postbus 9513, 2300 RA, Leiden, The Netherlands

${ }^{3}$ Hamburger Sternwarte, Universität Hamburg, Gojenbergsweg 112, 21029 Hamburg, Germany

${ }^{4}$ Centro de Astrobiología (CSIC-INTA), ESAC, Camino bajo del Castillo s/n, 28692 Villanueva de la Cañada, Madrid, Spain

*A full list of authors appears at the end of the abstract

Hydrodynamic escape is the most efficient atmospheric mechanism of planetary mass loss and has a large impact on planetary evolution. However, the lack of observations remained this mechanism poorly understood. Therefore, new observations of the He I triplet at $10830 \AA$ provide key information to advance hydrodynamic escape knowledge. In this work, we analyse the hydrodynamic escape of three exoplanets, HD209458 b, HD189733 b, and GJ 3470 b via an analysis of He triplet absorptions recently observed by the CARMENES high-resolution spectrograph, and their available Ly-alpha measurements, involving a 1D hydrodynamic model. We characterise the main upper atmospheric parameters, e.g., the temperature, the composition ( $\mathrm{H} / \mathrm{He}$ ratio), and the radial outflow velocity. We also study their hydrodynamic regime and show that HD209458 b is in the energy-limited regime, HD189733 b is in the recombination-limited regime, and G] 3470 b is in the photon-limited regime. Details of this work can be found in [1], [2], [3].

\section{References}

[1] Lampón, M., López-Puertas, M., Lara, L.M., et al. 2020, A\&A, 636, A13

[2] Lampón, M., López-Puertas, M., Sanz-Forcada, J., et al. 2021, A\&A, 647, A129

[3] Lampón, M., López-Puertas, M., Czesla, S., et al. 2021, A\&A, 648, L7

CARMENES Consortium: K. Molaverdikhani, A. Quirrenbach, E. Pallé, J. A. Caballero, Th. Henning, L. Nortmann, P. J. Amado, D. Montes, A. Reiners, and I. Ribas. 